\title{
Scapular kinematic alterations during arm elevation with decrease in pectoralis minor stiffness after stretching in healthy individuals
}

\section{$\operatorname{AUTHOR}(S):$}

Umehara, Jun; Nakamura, Masatoshi; Nishishita, Satoru; Tanaka, Hiroki; Kusano, Ken; Ichihashi, Noriaki

\section{CITATION:}

Umehara, Jun ...[et al]. Scapular kinematic alterations during arm elevation with decrease in pectoralis minor stiffness after stretching in healthy individuals. Journal of Shoulder and Elbow Surgery 2018, 27(7): 1214-1220

\section{ISSUE DATE:}

2018-07

URL:

http://hdl.handle.net/2433/244286

\section{RIGHT:}

(C) 2018. This manuscript version is made available under the CC-BY-NC-ND 4.0 license http://creativecommons.org/licenses/by-nc-nd/4.0/:; この論文は出版社版でありません 。引用の際には出版社版をご確認ご利用ください。; This is not the published version. Please cite only the published version. 
Scapular kinematics and pectoralis minor stiffness

\section{Title page}

2 Scapular kinematic alterations during arm elevation with decrease in pectoralis minor stiffness

3 after stretching in healthy individuals

5 Jun Umehara, MS. ${ }^{1 *}$, Masatoshi Nakamura, PhD. ${ }^{2)}$, Satoru Nishishita MS. ${ }^{1)}$, Hiroki Tanaka 6 MS. ${ }^{3)}$, Ken Kusano, MS. ${ }^{4}$, Noriaki Ichihashi, PhD. ${ }^{1)}$

1) Human Health Sciences, Graduate School of Medicine, Kyoto University, Kyoto, Japan

2) Institute for Human Movement and Medical Sciences, Niigata University of Health and Welfare, Niigata, Japan

3) Rehabilitation Unit, Kyoto University Hospital, Kyoto, Japan

4) ASICS Corporation, Institution of Sports Science, Kobe, Japan

\section{*Corresponding author}

Jun Umehara

Human Health Sciences, Graduate School of Medicine, Kyoto University, 53 Shogoin-Kawahawa-cho, Kyoto 606-8507, Japan

Phone: +81-75-751-3935; Fax: +81-75-751-3909

E-mail: umehara.jun.77z@st.kyoto-u.ac.jp

Financial biases: None

\section{Ethics committee approval}

The study design was approved by the ethic committee of Kyoto University Graduate School and the Faculty of Medicine (R0233). 
Scapular kinematics and pectoralis minor stiffness

\section{Comments}

27 Please publish the Figure 3 in color.

28

\section{Acknowledgement}

30 This study was supported by the Grant - in - Aid for Scientific Research (B) 15 H03043. 
Scapular kinematics and pectoralis minor stiffness

33

34

35

\section{Abstract}

Background: Pectoralis minor tightness may be seen in individuals with scapular dyskinesis, and stretching is used for the treatment of altered scapular motion in sports and clinical fields. However, few researchers have reported on the effects of the pectoralis minor stiffness on scapular motion during arm elevation. The purpose of this study is to investigate whether acute decrease of pectoralis minor stiffness after stretching changes the scapular motion during arm elevation.

Methods: Fifteen dominant and 15 non-dominant upper limbs in healthy men were allocated as control and interventional limbs, respectively. In the intervention limb group, the shoulder was passively and horizontally abducted at $150^{\circ}$ of elevation for five minutes to stretch the pectoralis minor muscle. Before and after stretching, three-dimensional scapular motion during abduction and scaption was examined using an electromagnetic sensor. Pectoralis minor stiffness was measured using ultrasonic shear wave elastography before and immediately after stretching, and after arm elevation.

Results: In the interventional limb, the pectoralis minor stiffness decreased by $3.2 \mathrm{kPa}$ immediately after stretching and $2.5 \mathrm{kPa}$ after arm elevation. The maximal changes in scapular kinematics after stretching were $4.8^{\circ}$ of external rotation and $3.3^{\circ}$ of posterior tilt in abduction, and $4.5^{\circ}$ of external rotation and $3.7^{\circ}$ of posterior tilt in scaption. No changes in upward rotation in abduction or scaption were seen.

Conclusion: Stretching for pectoralis minor muscle increases external rotation and posterior tilt of the scapula during arm elevation.

Level of evidence: Basic Science, Kinesiology Study.

Keywords: Shoulder; Physical therapy; Biomechanics; Stretching; Muscle stiffness; Elastography; Pectoralis minor muscle; 
Scapular kinematics and pectoralis minor stiffness

\section{Introduction}

The shoulder joint consists of the scapula, humerus, and clavicle, and is one of the largest and most complex joints in humans. The coordinated movement of these bones is important for optimal shoulder motion. Early authors investigating scapula motion in healthy individuals defined scapulohumeral rhythm, ${ }^{10}$ and it has been established that the scapula rotates upward, externally, and tilts posteriorly during arm elevation in healthy individuals. ${ }^{11,19,22}$ Additional researchers reported that scapular motion of the patients with impingement syndrome or glenohumeral instability was decreased in external and upward rotation and posterior tilt as compared with that of healthy individuals. ${ }^{2,17,21}$ Scapular dyskinesis has been defined as the set of abnormal motions and positions of scapula, ${ }^{14}$ and the evaluation and treatment for scapular dyskinesis may be essential for shoulder rehabilitation.

The onset scapular dyskinesis was related to the tightness of soft tissue surrounding the scapula. ${ }^{7,13}$ The tightness of the pectoralis minor muscle (PMi), ${ }^{7,13}$ the short head of the biceps brachii, ${ }^{13}$ the levator scapula, ${ }^{7}$ or the rhomboid ${ }^{7}$ has been speculated to cause scapular dyskinesis. Of these shoulder muscles, the PMi is the only muscle whose relationship between tightness and scapular dyskinesis has been verified by experimental study. Borstad et al. ${ }^{4}$ examined three-dimensional (3D) scapular motion during elevation in healthy individuals with and without a shortened PMi and showed that a decrease in external rotation and posterior tilt are seen in individuals with a shortened PMi. The altered scapular kinematics, which is found in subjects with shortened PMi, seen in this previous study ${ }^{4}$ was similar to that observed in many patients with shoulder disease. ${ }^{15,17}$ Therefore, PMi tension may be important factor in scapular dyskinesis.

Stretching is applied as an approach to scapular dyskinesis caused by the PMi tightness.

Borstad et al. ${ }^{3}$ recommended a unilateral corner stretch as one self-stretch method for the PMi. Umehara et al. ${ }^{31}$ also showed that shoulder horizontal abduction at an elevation of $150^{\circ}$ was the 
Scapular kinematics and pectoralis minor stiffness

84 most effective stretching technique for the PMi. Considering that there is a correlation between

85 PMi stiffness and scapular dyskinesis, it is obvious that investigating not only the stretching maneuver but also the change in the PMi stiffness and scapular motion after stretching is important. However, little is examined on this relationship. stiffness after stretching alters the 3D scapular motion during arm elevation. Borstad et al. ${ }^{4}$ reported a decrease in external rotation and posterior tilt of scapula in individuals with a shortened PMi as compared with in healthy individuals. Therefore, we hypothesized that the decrease in PMi stiffness after stretching augments the external rotation and posterior tilt of

93 scapula during arm elevation. 
Scapular kinematics and pectoralis minor stiffness

\section{Materials and Methods}

\section{Participants}

This study was a controlled experimental study. Twenty men (age, 25.4 \pm 3.1 years; height, $171.5 \pm 5.3 \mathrm{~cm}$; weight, $67.6 \pm 8.5 \mathrm{~kg}$ ) participated in this study. Dominant and non-dominant upper limbs were allocated as control and interventional limbs respectively. The subjects were randomly recruited from the students at our institution. Upon selection, the subjects orally confirmed that they do not meet the exclusion criteria, which included female gender, designation as an athlete or performing any extensive exercise, a history of orthopedic or nervous system disease in upper limb. Considering that the low body mass index minimized skin motion artifacts in the measurement of scapular motion during arm elevation, we also excluded the subject with body mass index $>25$, calculated using the height and weight. Prior to the experiment, four men —one with a daily extensive exercise regimen, one with a history of shoulder pain, and two with a high body mass index-were excluded. The aim and procedures of the study were explained to all subjects, and informed consent was obtained. The study protocol was approved by the ethics committee of our institution, and conformed the principle of the Declaration of Helsinki.

\section{Experimental Procedures}

The participants, while sitting on a wooden stool, performed shoulder abduction (elevation in the coronal plane) and scaption (elevation in the scapular plane) before and after the PMi stretching. The stretching procedure of the PMi is described in detail in our previous study (Figure 1). ${ }^{31}$ The participants underwent stretching to the point of discomfort (but not pain) for five minutes (30 seconds, 10 repetitions, 10-second intervals). Each elevation plane was marked on the floor using sections of elastic tape. In the starting posture, keeping the upper limb aside the body with the elbow fully extended, the palm facing the body, and the eyes looking straight 
Scapular kinematics and pectoralis minor stiffness familiarization to the abduction and scaption before the assessment.

\section{Instrumentation}

\section{Scapular Kinematics}

forward on the target at eye height, the participant was asked to raise their arm to full elevation in four seconds and then lower it to starting position in four seconds three times consecutively to the rhythm of a metronome with 60 BPM. The participant underwent sufficient

The 3D motion of the shoulder complex during arm elevation before and after stretching was measured using an electromagnetic tracking device (Liberty; Polhemus, Colchester, VT, USA) at $120 \mathrm{~Hz}$. This system consists of a transmitter, five sensors, and a digitizing stylus operated by an electronic unit. The transmitter was fixed on a rigid wooden board at a height of $40 \mathrm{~cm}$ from the floor and $30 \mathrm{~cm}$ behind the subjects. An electromagnetic field was generated by the transmitter, and was sensed by these sensors and the stylus. This electromagnetic field represented the global coordinate system, with the $\mathrm{X}$-axis pointing forward, the Y-axis pointing upward, the Z-axis pointing to the right, and the origin located at the transmitter. Next, the sensors were attached to the bony landmarks of the subjects with adhesive tape. The thoracic sensor was placed on the sternum just inferior to the jugular notch, the humeral sensor was placed on the middle point of the humerus with a thermoplastic cuff, and the scapular sensor was placed on the flat surface of acromion. Based on these sensors' placement, the local coordinate system of the thorax, humerus, and scapula were established by digitizing each bony landmark. All definitions of the local coordinate system were in accordance with the shoulder standardization proposal of the International Society of Biomechanics, ${ }^{33}$ and the glenohumeral rotation center in the humeral segment was defined with reference to the previous study. ${ }^{23}$ The rotation of the distal coordinate system was described with respect to the proximal coordinate system, according to the Euler angle of the International Society of Biomechanics. ${ }^{33}$ 
Scapular kinematics and pectoralis minor stiffness

To describe the joint motion in correspondence with human kinesiology, the motion of the scapula around the Ys-axis was defined as internal rotation (positive) and external rotation (negative); the motion around the Xs-axis was defined as downward rotation (positive) and upward rotation (negative); the motion around Zs-axis was defined as posterior tilt (positive) and anterior tilt (negative); and the motion of the humerus around Xh-axis was defined as elevation (positive) (Figure 2). These motions were calculated using MATLAB (The Math Works, Natick, MA, USA). The scapular rotation was measured in every $10^{\circ}$ of humeral elevation relative to the thorax, from $30^{\circ}$ to $120^{\circ}$ of humeral elevation. These angles was selected because the previous study ${ }^{12}$ reported that there was little influence of the artifact of soft tissue on measuring the scapular motion in humeral elevations of less than $120^{\circ}$ using a surface method. The elevation was examined three times, and the mean value was used for analysis.

\section{Muscle Stiffness}

The PMi stiffness was measured before stretching, immediately after stretching, and after arm elevation using ultrasonic shear wave elastography (Aixplorer, SuperSonic Imagine, Aix-enProvence, France) with an ultrasound transducer (SL15-4: 4 to $15 \mathrm{MHz}$ linear probe) (Figure 3). The ultrasonic shear wave elastography monitors the propagation of shear waves generated in tissue using acoustic radiation forces, and is able to evaluate the tissue elasticity of individual muscles. ${ }^{28}$ The shear elastic modulus of the muscle represents muscle stiffness, and has been used as a quantitative indicator of the stretching effect in many previous studies. ${ }^{24,29,34}$ The shear elastic modulus $(G)$ was calculated from the shear wave propagation speed (V) generated by the transducer using the formula of $G=\rho V^{2}$, in which $\rho$ is the muscle density $\left(1,000 \mathrm{~kg} / \mathrm{m}^{3}\right)$. The validity of applying the shear wave elastography to evaluate the skeletal muscle was reported in a previous study. ${ }^{8}$ The stiffness was measured three times in each session, and the 
Scapular kinematics and pectoralis minor stiffness

170

171

172

173

mean value was used for analysis. All calculation of the stiffness was blinded by anonymizing the ultrasonic image, and a region of interest of the shear modulus was carefully chosen as large as possible with exclusion of subcutaneous adipose tissues and aponeuroses.

For the reliability study, the PMi stiffness was measured in ten healthy men (age, $24.9 \pm 1.5$ years; height, $171.7 \pm 6.5 . \mathrm{cm}$; weight, $70.4 \pm 7.8 \mathrm{~kg}$ ) prior to this study. Following the completion of the measurement method mentioned above, the stiffness was measured three times with sufficient rest interval. The intra-observer reliability of the ultrasonic measurement was confirmed using the intraclass correlation coefficient $(1,3)\left(\mathrm{ICC}_{1,3}\right)$ with $95 \%$ confidence interval (95\% CI). ICC 1,3 was 0.99 (95\% CI: 0.97-0.99). A previous study investigating the reliability coefficient reported that a range from 0.81 to 1.00 was "almost perfect" reproducibility. ${ }^{16}$ The measurement of PMi stiffness in our study, therefore, was considered to be reproducible.

\section{Data analysis}

Only those subjects whose decrease in muscle stiffness remained until after arm elevation were analyzed in this study. We focused on the effect of the decrease in PMi stiffness on the scapular motion but not the PMi stretching, so that we could examine the direct relationship between the PMi stiffness and scapular motion. Statistical analysis was performed with IBM SPSS Statistical software (version 22; IBM, Armonk, NY, USA).

Regarding the stiffness in raw data before stretching, a paired $t$-test was performed to compare the interventional and the control limbs. The amount of change in the stiffness was calculated by subtracting the value of the stiffness before stretching from that present immediately after stretching or after elevation. For the change in stiffness, a two-way analysis of variance (ANOVA) with repeated measures on two factors [limb (two levels, interventional limb; control limb) $\times$ time (two levels, immediately after stretching - before stretching; after 
Scapular kinematics and pectoralis minor stiffness

195

196

197

198

199

200

201

202

203

204

205

206

207

208

209

210

211

212

arm elevation - before stretching)] was used to demonstrate that the stretching decreased the PMi stiffness. If a significant main effect was found, then a Bonferroni post hoc test was performed. A confidence level of .05 was used in all statistics tests. Cohen's $d$ values were also reported as the effect size, with the values of $0.2,0.5$, and 0.8 considered to elicit small, moderate, and large effects, respectively. ${ }^{6}$

The amount of change in the scapular motion was calculated by subtracting the value of scapular motion before stretching from that of it after stretching. For the change in each scapular motion (i.e. internal/external rotation; downward/upward rotation; posterior/anterior tilt), a two-way ANOVA with repeated measures on two factors [limb (two levels, interventional limb; control limb) $\times$ angle (ten levels, every $10^{\circ}$ from $30^{\circ}$ to $120^{\circ}$ )] was used to determine the effects of the change in PMi stiffness on the scapular motion during elevation. If a significant interaction was found, then a paired $t$-test for post hoc test was performed to compare the interventional limb with the control limb with respect to each angle. If a significant main effect of the side was also found, then a Bonferroni post hoc test was performed to compare the interventional limb with the control limb. The significant main effect of the angle was ignored because the present study was interested in the comparison between the interventional limb and the control limb. 
Scapular kinematics and pectoralis minor stiffness

213

214

215

216

\section{Results}

In the dominant limb, the decrease in the PMi stiffness immediately after stretching and after

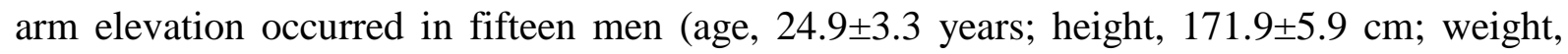
$67.2 \pm 8.4 \mathrm{~kg}$ ); therefore, the results of these fifteen men (thirty shoulders) are shown below.

The paired $t$-test showed no significant differences between the interventional limb and the control limb in stiffness before stretching ( $\mathrm{P}=.063,95 \% \mathrm{IC}:-0.24-7.98)$. For the amount of change in the stiffness, a two-way ANOVA indicated a significant main effect of the limb but not the time, with no significant interaction between the limb and the time (Table 1).

The raw value and the amount of change in scapular motion for abduction are shown in Table 2. For the amount of change in the internal/external rotation of scapula, a two-way ANOVA showed a significant interaction between the limb and the angle $(F=4.519, P=.029)$. Then, a post hoc test indicated that the change in the interventional limb was significantly greater than that in the control limb from $40^{\circ}$ to $120^{\circ}$ abduction $(\mathrm{P}=.001-.014)$, and an increase in external rotation in interventional limb was found after stretching. For the amount of change in the downward/upward rotation of the scapula, there were no significant interactions between the limb and the angle $(\mathrm{F}=0.345, \mathrm{P}=.726)$, and no significant main effect in the limb $(\mathrm{F}=$ $0.129, \mathrm{P}=.725)$. For the amount of change in the posterior/anterior tilt of scapula, a two-way ANOVA showed no significant interaction between the limb and the angle $(\mathrm{F}=0.891, \mathrm{P}=.378)$, but a significant main effect in the limb was seen $(F=4.966, P=.043)$. A post hoc test indicated that the change in the interventional limb was significantly greater than that in the control limb $(\mathrm{P}=.043)$, and that the posterior tilt in the interventional limb increased after stretching throughout the full evaluated range.

The raw value and the amount of change in scapular motion for scaption are shown in Table 3. For the amount of change in the internal/external rotation of scapula, a two-way ANOVA showed a significant interaction between the limb and the angle $(F=6.655, P=.004)$. 
Scapular kinematics and pectoralis minor stiffness

238 Then, a post hoc test indicated that the amount of change from $40^{\circ}$ to $120^{\circ}$ in the interventional

239 limb was significantly greater than that in the control limb $(\mathrm{P}=.001-.034)$, and that the external

240 rotation in the interventional limb increased after stretching. For the amount of change in the

241 downward/upward rotation of scapula, there were no significant interactions between the limb

242 and the angle $(\mathrm{F}=0.295, \mathrm{P}=.750)$, and no significant main effect in the limb $(\mathrm{F}=0.006, \mathrm{P}$

$243=.940$ ). For the amount of change in the posterior/anterior tilt of scapula, a two-way ANOVA

244 showed a significant interaction between the limb and the angle $(\mathrm{F}=4.397, \mathrm{P}=.032)$. Then, a

245 post hoc test indicated that the amount of change from $50^{\circ}$ to $120^{\circ}$ in the interventional limb

246 was significantly greater than in the control limb $(\mathrm{P}=.006-.035)$, and the posterior tilt in the

247 interventional limb increased after stretching. 
Scapular kinematics and pectoralis minor stiffness

\section{Discussion}

The present study investigated the effects of PMi stiffness on 3D scapular motion during arm elevation, and found a decrease in PMi stiffness and an increase in external rotation and posterior tilt of the scapula after stretching. These results indicate that the alteration in scapular motion in combination with decrease in PMi stiffness occurred after stretching, and accorded with our hypothesis. To the best of our knowledge, this is the first study to demonstrate that an acute decrease in PMi stiffness after stretching changes the 3D scapular motion during arm elevation.

The decrease in the PMi stiffness occurred immediately after stretching and lasted until after arm elevation. The previous study ${ }^{24}$ showed a positive correlation between the rate of change in the shear elastic modulus and the rate of change in muscle stiffness, and therefore, the decrease seen in shear elastic modulus after stretching indicates a decrease in muscle stiffness (a so-called increase in the muscle flexibility). ${ }^{1,9,30,34}$ Therefore, the decrease in the PMi stiffness immediately after stretching and after arm elevation confirmed that the stretching used in the current study was sufficient in decreasing PMi stiffness until the end of the evaluation.

To the best of our knowledge, there is only one study that investigated the relationship between the acute change in the flexibility of PMi and 3D scapular motion. Williams et al. ${ }^{32}$ measured the PMi length (coracoid process to forth rib) and the scapular kinematics before and after two types of stretching, focused stretch or gross stretch, for one minute (30 seconds, two repetitions, 30-second intervals). They ${ }^{32}$ concluded that there are no changes in the scapular kinematics after either form of stretching, which was inconsistent with our results, which noted that a change in scapular motion occurred after PMi stretching. This discrepancy between the previous study and our results could be attributed to the duration time and the index of stretching effect. Among various studies on stretching duration, there is a previous study ${ }^{25}$ that 
Scapular kinematics and pectoralis minor stiffness

274

275

276

277

examined the minimum time required for stretching to change the passive property. Nakamura et al. ${ }^{25}$ concluded that stretching for more than two minutes was recommended to decrease the passive property of the gastrocnemius muscle. Therefore, though it is necessary to consider the difference in the muscles studied, it is possible that the stretching duration of the PMi was insufficient to elicit a change in scapular kinematics in Williams's study. Furthermore, there is also a possibility the length of the PMi may not be sufficient to represent PMi flexibility due to the bias of skins, soft tissues, and/or posture. In contrast, we measured the PMi stiffness quantitatively using the shear elastic modulus measured by ultrasonic shear wave elastography. Therefore, our study might be more valid than their study in investigating the relation of the PMi stiffness and the scapular motion.

These results mean that the decrease in the PMi stiffness after stretching induced the external rotation and posterior tilt of scapula. Generally, the scapula rotates upward and externally and tilts posteriorly during arm elevation in healthy individuals. ${ }^{12,18,22}$ The PMi tightness might cause the internal and downward rotation and anterior tilt of scapula from an anatomic perspective. ${ }^{5,20,26}$ Borstad et al. ${ }^{4}$ showed that the external rotation and posterior tilt in individuals with shortened PMi muscles were decreased when compared with individuals with long PMi during arm elevation. Therefore, considering these studies, our results suggested that the scapular external rotation and posterior tilt increased in abduction and scaption with a decrease in PMi stiffness. For the posterior tilt in the interventional limb group after stretching, there were differences in the amount of change between abduction and scaption. These differences in behavior, which the increase in the posterior tilt and occurred from $30^{\circ}$ to $120^{\circ}$ in abduction and from $50^{\circ}$ to $120^{\circ}$ in scaption, might depend on the difference in the plane of elevation. Compared with scaption, abduction needs a slightly greater posterior tilt of the scapula, due to the difference of the plane although there are no significant differences. ${ }^{19}$ Therefore, it is rational that the posterior tilt of the scapula may arise from the early phase of 
Scapular kinematics and pectoralis minor stiffness abduction by the decrease in PMi stiffness.

The maximum amount of change in external rotation and the posterior tilt were $4.8^{\circ}$ and $3.3^{\circ}$ in abduction, and $4.5^{\circ}$ and $3.7^{\circ}$ in scaption. Ludewig and Cook $^{17}$ reported that individuals with shoulder impingement had increased internal rotation, decreased upward rotation, and decreased posterior tilt during arm elevation. The previous ${ }^{17}$ study suggested that $4^{\circ}$ to $6^{\circ}$ of change in scapular motion is important in narrowing of the subacromial space and the occurrence of impingement, because individuals with impingement syndrome showed increased internal rotation of $5.2^{\circ}$, decreased upward rotation of $4.1^{\circ}$, and increased anterior title of $5.8^{\circ}$ as compared with healthy individuals. We believe that our findings are of clinical relevance because the change in scapular motion after stretching shown in the present study approximately correspond with the range of changes seen in scapular motion between healthy individuals and those with impingement syndrome indicated in Ludewig and Cook's study. The change in the scapular motion after the stretching observed in the current study may be of clinical significance; however, further research is warranted to validate these theories within pathologic subjects.

However, when interpreting our findings, one should note the following: first, the subjects were all healthy men, as prescribed by the exclusion criteria. Therefore, it is unclear whether the findings can be generalized to individuals with impingement syndrome. Second, the stiffness of only the PMi was measured among the shoulder girdle muscles. Therefore, this study does not exactly promise that only a decrease in the PMi stiffness changed the scapular kinematics, and it is possible that other muscles such as the pectoralis major muscle, the subscapularis muscle, or glenohumeral ligaments and capsules were also stretched and thus had an effect. This is the limitation of a in-vivo study. Third, the current study investigated only the acute effect of the PMi stretching on the scapular motion, so its long-term effect is unknown. The recent study examining the effects of self-stretching of the PMi for six weeks on the 
Scapular kinematics and pectoralis minor stiffness

324 scapular kinematics concluded that stretching did not change PMi length and scapular

325 kinematics in individuals with and without shoulder pain. ${ }^{27}$ Therefore, future study should 326 evaluate the long-term effects of the therapist-applied PMi stretching on muscle stiffness and 327 scapular motion. 
Scapular kinematics and pectoralis minor stiffness

\section{Conclusion}

330 We investigated the effects of PMi stiffness after stretching on the change in scapular motion

331 during arm elevation. Our results indicated a decrease in PMi stiffness, increased external 332 rotation and posterior tilt of the scapula occurred during arm elevation after stretching. These

333 findings might be relevant knowledge for the approach to scapular dyskinesis and in further 334 studies.

335 
Scapular kinematics and pectoralis minor stiffness

336

337

338

339

\section{References}

1. Akagi R, Takahashi H. Acute effect of static stretching on hardness of the gastrocnemius muscle. Med Sci Sports Exerc 2013;45(7):1348-1354. doi:10.1249/MSS.0b013e3182850e17.

2. Borstad JD, Ludewig PM. Comparison of scapular kinematics between elevation and lowering of the arm in the scapular plane. Clin Biomech 2002;17:650-659. doi:10.1016/S0268-0033(02)00136-5.

3. Borstad JD, Ludewig PM. Comparison of three stretches for the pectoralis minor muscle. J Shoulder Elbow Surg 2006;15(3):324-330. doi:10.1016/j.jse.2005.08.011.

4. Borstad JD, Ludewig PM. The effect of long versus short pectoralis minor resting length on scapular kinematics in healthy individuals. J Orthop Sports Phys Ther 2005;35(4):227-238. doi:10.2519/jospt.2005.1669.

5. Burkhart SS, Morgan CD, Ben Kibler W. The disabled throwing shoulder: Spectrum of pathology Part I: Pathoanatomy and biomechanics. Arthrosc - J Arthrosc Relat Surg 2003;19(4):404-420. doi:10.1053/jars.2003.50128.

6. Cohen J. Statistical Power Analysis. Current Directions in Psychological Science 1992; 1(3):98-101

7. Cools AMJ, Struyf F, De Mey K, Maenhout A, Castelein B, Cagnie B. Rehabilitation of scapular dyskinesis: from the office worker to the elite overhead athlete. Br J Sports Med 2014;48(8):692-697. doi:10.1136/bjsports-2013-092148.

8. $\quad$ Eby SF, Song P, Chen S, Chen Q, Greenleaf JF, An KN. Validation of shear wave elastography in skeletal muscle. J Biomech 2013;46(14):2381-2387. doi: 10.1016/j.jbiomech.2013.07.033

9. Ichihashi N, Umegaki H, Ikezoe T, Nakamura M, Nishishita S, Fujita K, et al. The effects of a 4-week static stretching programme on the individual muscles 
Scapular kinematics and pectoralis minor stiffness

361

362

363

364

365

366

367

368

369

370

371

372

373

374

375

376

377

378

379

380

381

382

383

384

385 comprising the hamstrings. J Sports Sci 2016;34(23):2155-2159. doi:10.1080/02640414.2016.1172725.

10. Inman VT, Saunders FRCS, Abbott LC. Observations on the function of the shoulder joint. J Bone Jt Surg Am 1944;26(1):1-30.

11. Johnson GR, Stuart PR, Mitchell S. A method for the measurement of threedimensional scapular movement. Clin Biomech 1993;8(5):269-273. doi:10.1016/02680033(93)90037-I.

12. Karduna AR, McClure PW, Michener LA, Sennett B. Dynamic measurements of three-dimensional scapular kinematics: a validation study. J Biomech Eng 2001;123(2):184-190. doi:10.1115/1.1351892.

13. Kibler WB, Ludewig PM, McClure PW, Michener LA, Bak K, Sciascia AD. Clinical implications of scapular dyskinesis in shoulder injury: the 2013 consensus statement from the “Scapular Summit”. Br J Sports Med 2013;47(14):877-885. doi:10.1136/bjsports-2013-092425.

14. Kibler WB. The Role of the Scapula in Athletic Shoulder Function. Am J Sports Med 1998:26(2):325-337. doi:10.1177/03635465980260022801

15. Kijima T, Matsuki K, Ochiai N, Yamaguchi T, Sasaki Y, Hashimoto E, et al. In vivo 3-dimensional analysis of scapular and glenohumeral kinematics: comparison of symptomatic or asymptomatic shoulders with rotator cuff tears and healthy

shoulders. $\quad$ J Shoulder Elb Surg 2015;24(11):1817-1826. doi:10.1016/j.jse.2015.06.003.

16. Landis JR, Koch GG. The measurement of observer agreement for categorical data. Biometrics 1977;33(1):159-174.

17. Ludewig PM, Cook TM. Alterations in shoulder kinematics and associated muscle activity in people with symptoms of shoulder impingement. Phys Ther 2000;80(3):276-291. 
Scapular kinematics and pectoralis minor stiffness

386

387

388

389

390

391

392

393

394

395

396

397

398

399

400

401

402

403

404

405

406

407

408

409

410

18. Ludewig PM, Cook TM, Nawoczenski D a. Three-dimensional scapular orientation and muscle activity at selected positions of humeral elevation. J Orthop Sports Phys Ther 1996;24(2):57-65. doi:10.2519/jospt.1996.24.2.57.

19. Ludewig PM, Phadke V, Braman JP, Hassett DR, Cieminski CJ, LaPrade RF. Motion of the shoulder complex during multiplanar humeral elevation. J Bone Joint Surg Am 2009;91(2):378-389. doi:10.2106/JBJS.G.01483.

20. Ludewig PM, Reynolds JF. The association of scapular kinematics and glenohumeral joint pathologies. J Orthop Sports Phys Ther 2009;39(2):90-104.

doi:10.2519/jospt.2009.2808.

21. Matias R, Pascoal AG. The unstable shoulder in arm elevation: A three-dimensional and electromyographic study in subjects with glenohumeral instability. Clin Biomech 2006;21:S52-S58. doi:10.1016/j.clinbiomech.2005.09.014.

22. McClure PW, Michener LA, Sennett BJ, Karduna AR. Direct 3-dimensional measurement of scapular kinematics during dynamic movements in vivo. J Shoulder Elb Surg 2001;10(3):269- 277. doi:10.1067/mse.2001.112954.

23. Murray IA. Determining upper limb kinematics and dynamics during everyday tasks. PhD Thesis, Cent Rehabil Eng Stud Univ Newcastle upon Tyne 1999;1-470. https://theses.ncl.ac.uk/dspace/bitstream/10443/185/1/murray99.pdf.

24. Nakamura M, Ikezoe T, Kobayashi T, Umegaki H, Takeno Y, Nishishita S, et al. Acute effects of static stretching on muscle hardness of the medial gastrocnemius muscle belly in humans: An ultrasonic shear-wave elastography study. Ultrasound Med Biol 2014;40(9):1991-1997. doi:10.1016/j.ultrasmedbio.2014.03.024.

25. Nakamura M, Ikezoe T, Takeno Y, Ichihashi N. Time course of changes in passive properties of the gastrocnemius muscle-tendon unit during 5 min of static stretching. Man Ther 2013;18(3):211-215. doi:10.1016/j.math.2012.09.010. 
Scapular kinematics and pectoralis minor stiffness

26. Novak CB, Mackinnon SE. Repetitive use and static postures: A source of nerve compression and pain. J Hand Ther 1997;10(2):151-159. ～doi:10.1016/S08941130(97)80069-5.

27. Rosa DP, Borstad JD, Pogetti LS, Camargo PR. Effects of a stretching protocol for the pectoralis minor on muscle length, function, and scapular kinematics in individuals with and without shoulder pain. J Hand Ther 2017;30(1):20-29. doi:10.1016/j.jht.2016.06.006.

28. Shiina T, Nightingale KR, Palmeri ML, et al. WFUMB guidelines and recommendations for clinical use of ultrasound elastography: Part 1: basic principles and terminology. Ultrasound Med Biol 2015;41(5):1126-1147. doi:10.1016/j.ultrasmedbio.2015.03.009.

29. Taniguchi K, Shinohara M, Nozaki S, Katayose M. Acute decrease in the stiffness of resting muscle belly due to static stretching. Scand J Med Sci Sports 2015;25(1):32-

40. doi:10.1111/sms.12146.

30. Umehara J, Hasegawa S, Nakamura M, Nishishita S, Umegaki H, Tanaka H, et al. Effect of scapular stabilization during cross-body stretch on the hardness of infraspinatus, teres minor, and deltoid muscles: An ultrasonic shear wave elastography study. Musculoskelet Sci Pract 2017;27:91-96. doi:10.1016/j.math.2016.10.004.

31. Umehara J, Nakamura M, Fujita K, Kusano K, Nishishita S, Araki K, et al. Shoulder horizontal abduction stretching effectively increases shear elastic modulus of pectoralis minor muscle. J Shoulder Elb Surg 2017; 26(7):1159-1165. doi:10.1016/j.jse.2016.12.074.

32. Williams JG, Laudner KG, McLoda T. The acute effects of two passive stretch maneuvers on pectoralis minor length and scapular kinematics among collegiate 
Scapular kinematics and pectoralis minor stiffness

436

437

438

439

440

441

442

443

444

445 swimmers. Int J Sports Phys Ther 2013;8(1):25-33.

33. Wu G, van der Helm FC, Veeger HE, Makhsous M, Van Roy P, Anglin C, et al. ISB recommendation on definitions of joint coordinate systems of various joints for the reporting of human joint motion-Part II: shoulder, elbow, wrist and hand. J Biomech 2005;38(5):981-992. doi:10.1016/j.jbiomech.2004.05.042.

34. Yamauchi T, Hasegawa S, Nakamura M, Nishishita S, Yanase K, Fujita K, et al. Effects of two stretching methods on shoulder range of motion and muscle stiffness in baseball players with posterior shoulder tightness: a randomized controlled trial. J shoulder Elb Surg 2016;25(9):1395-1403. doi:10.1016/j.jse.2016.04.025. 
Scapular kinematics and pectoralis minor stiffness

\section{Figure and Table Legends}

Figure 1; Stretching of the pectoralis minor muscle. As directed, the subject sat on the wooden stool and the interventional limb was brought to maximal horizontal abduction and external rotation at an arm elevation of $150^{\circ}$ with the elbow in $90^{\circ}$ flexion, and was subsequently maximally externally rotated by the investigator. During the stretching, the participants was instructed to remain relaxed. The investigator operated the upper limb of the subject using one hand and held the trunk using the other hand.

Figure 2; The definition of coordinate systems and motions relative to the thorax for the scapula. The scapula are seen in the posterior view of the right shoulder.

Figure 3; Posture and measurement site of the pectoralis minor stiffness are shown in A. The participant sat on a wooden stool with their arm relaxed on a platform in a position with $90^{\circ}$ of shoulder abduction and $90^{\circ}$ of elbow flexion. The measurement site was defied as the midpoint between the coracoid process and the forth rib-sternum junction. The probe was placed parallel to the muscle fascicle of the PMi on the ultrasonic image as B. The participant was instructed to hold their breath during measurement to prevent elongation of the PMi due to the motion of rib cage. PMa, pectoralis major muscle; PMi, pectoralis minor muscle; IM, intercostal muscle.

Table 1; Pectoralis minor muscle stiffness $(\mathrm{kPa})$ pre-assessment and post assessment.

Table 2; Raw value and amount of change in scapular motion for abduction.

Table 3; Raw value and amount of change in scapular motion for scaption. 
Scapular kinematics and pectoralis minor stiffness

$471 \quad$ Figure 1

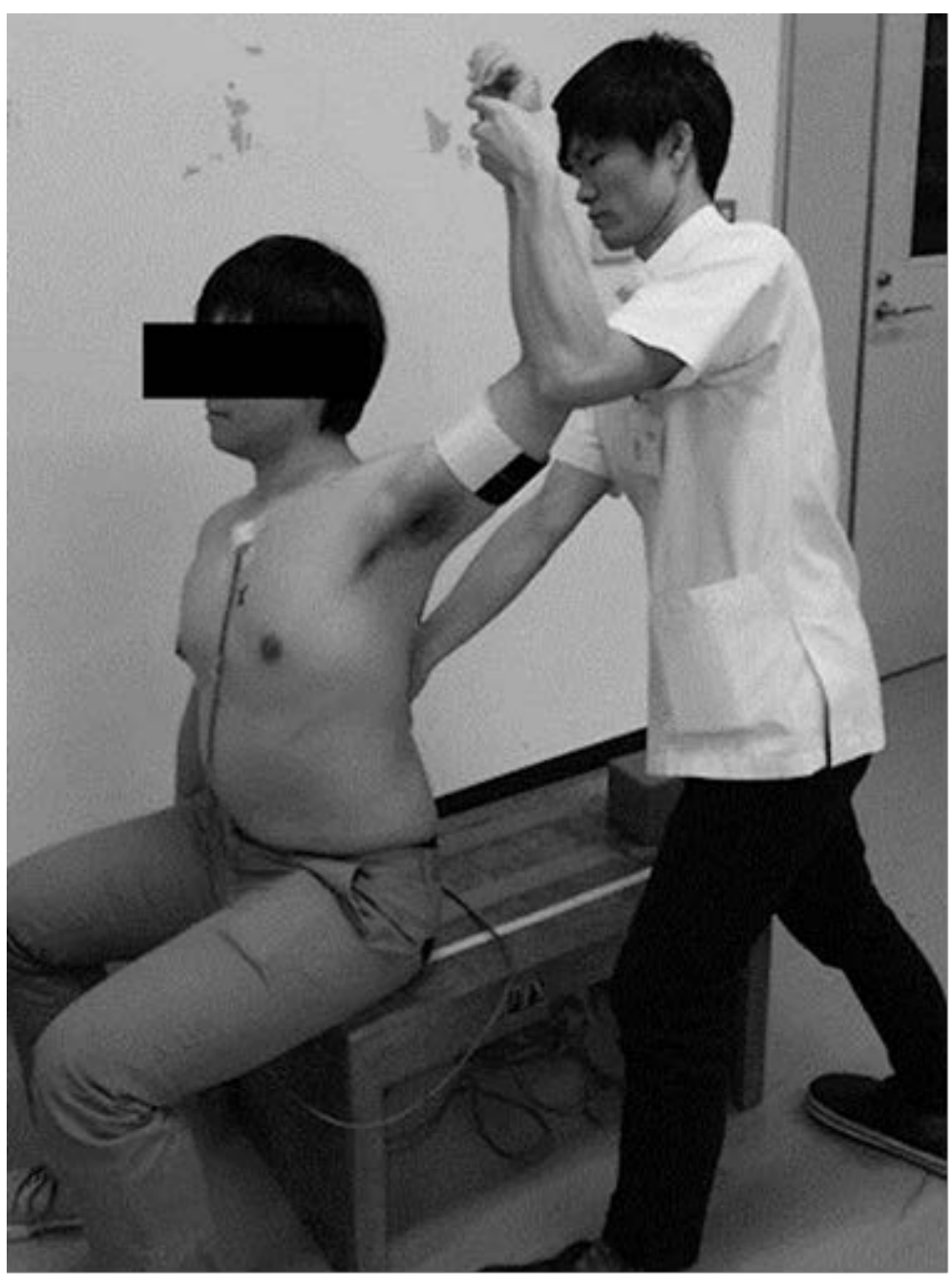

472 
Scapular kinematics and pectoralis minor stiffness

$474 \quad$ Figure 2

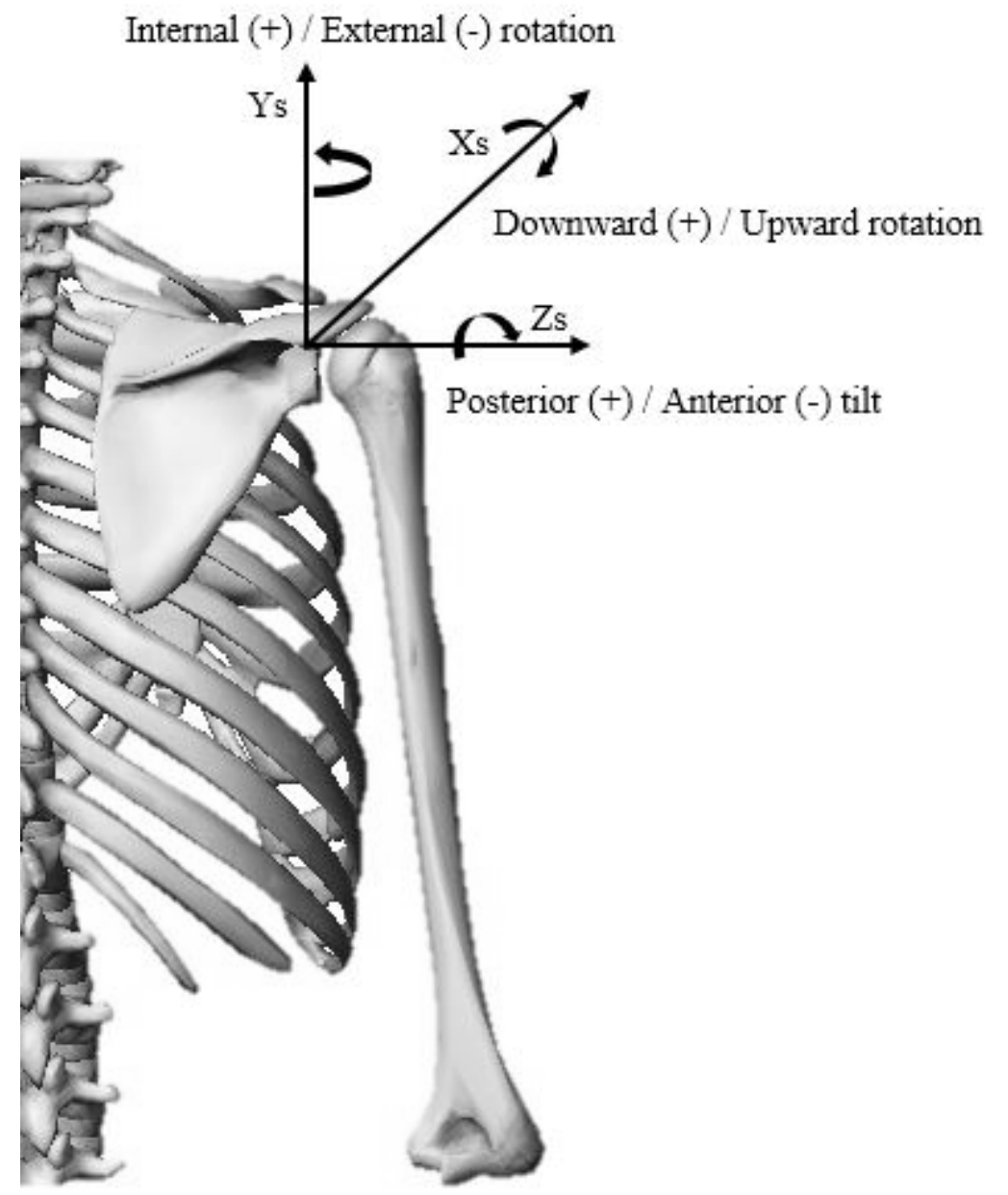


Scapular kinematics and pectoralis minor stiffness

$478 \quad$ Figure 3

479

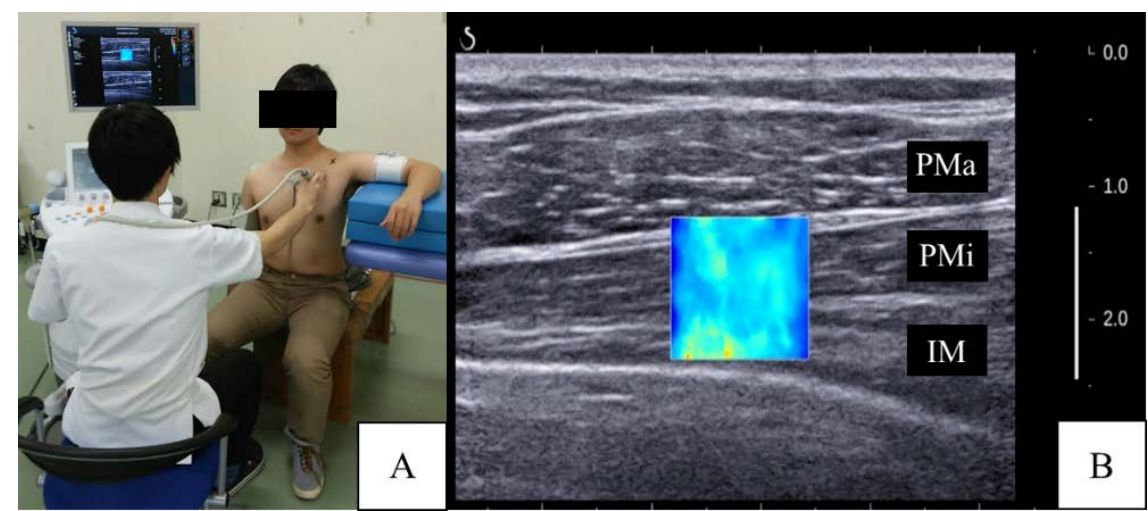

480 
Scapular kinematics and pectoralis minor stiffness

481 Table 1

482

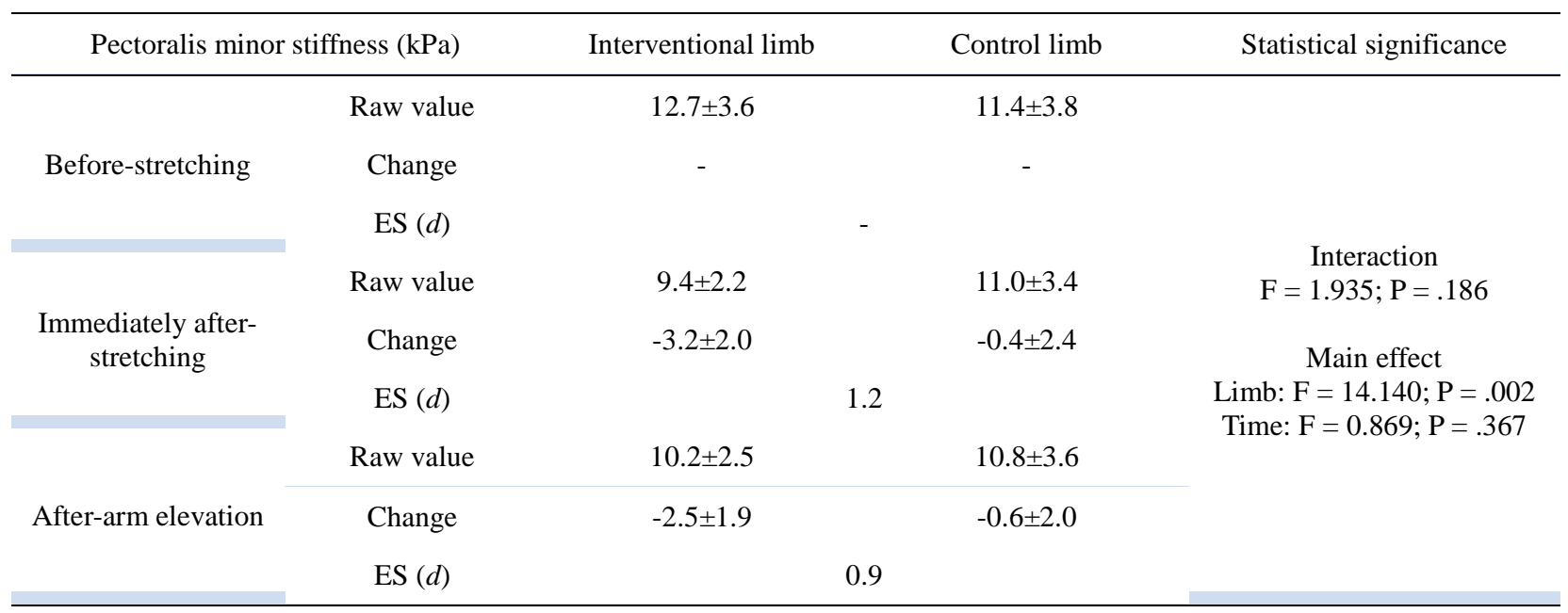

483

484

485

486

487
Change, amount of change between before and after stretching, and before and after arm elevation. Value \pm standard deviation; ES, effect size. 
Scapular kinematics and pectoralis minor stiffness

489

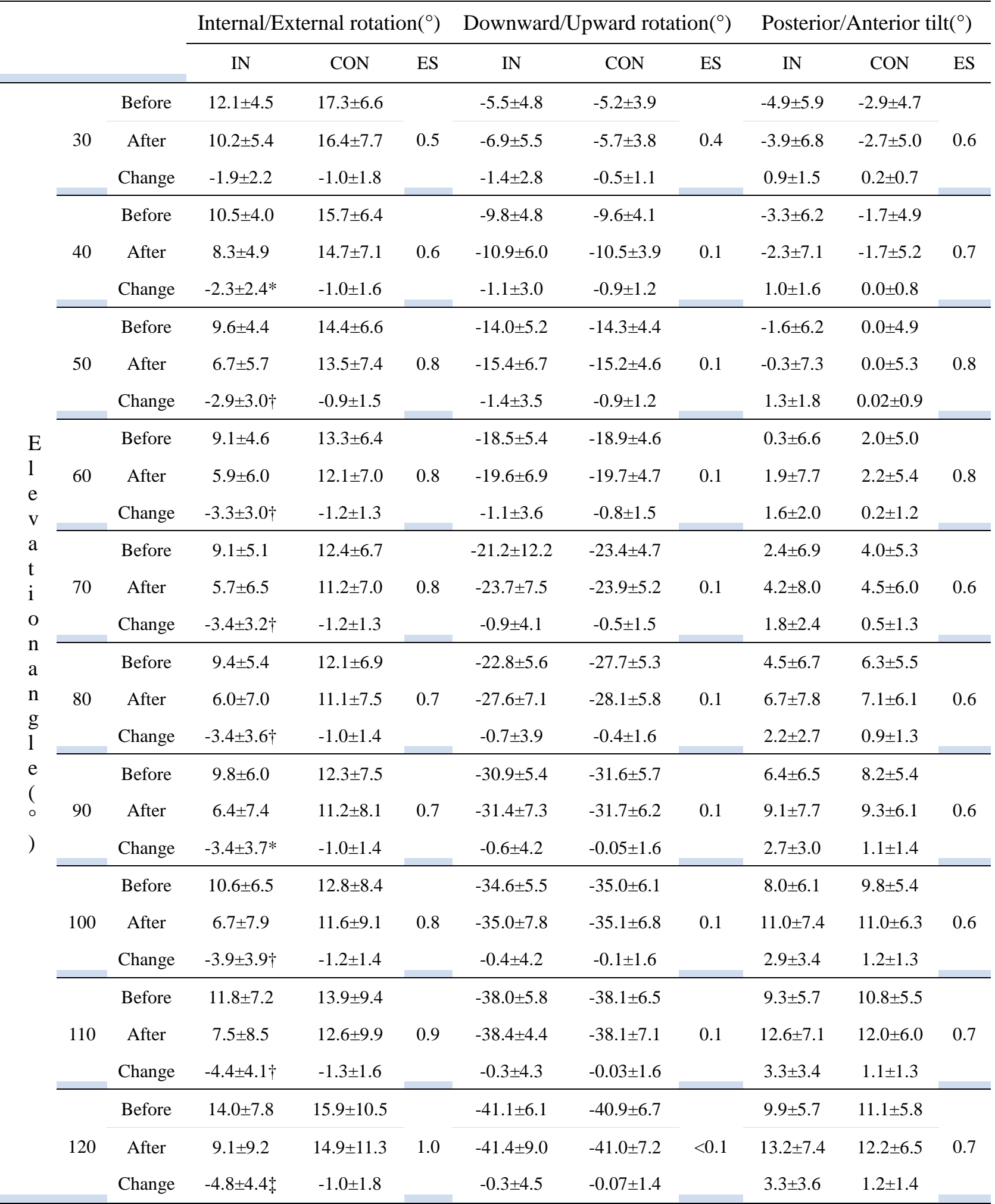

490
IN, interventional limb; CON, control limb; ES, effect size of amount of change; Before, raw value before stretching; After, raw value after stretching; Change, amount of change between before and after stretching. Values are expressed as mean \pm standard deviation. The asterisk indicates that the change in scapular motion in the interventional limb is significantly $(P<.05)$ greater than it in the control limb; single dagger indicates that it in the interventional limb is significantly $(\mathrm{P}<.01)$ greater than it in the control limb; and double dagger 
Scapular kinematics and pectoralis minor stiffness

496

indicates that it in the interventional limb is significantly $(\mathrm{P}<.001)$ greater than it in the control limb.

498 
Scapular kinematics and pectoralis minor stiffness

499

500

Table 3

\begin{tabular}{|c|c|c|c|c|c|c|c|c|c|c|c|}
\hline & & & \multicolumn{3}{|c|}{ Internal/External rotation $\left({ }^{\circ}\right)$} & \multicolumn{3}{|c|}{ Downward/Upward rotation $\left(^{\circ}\right)$} & \multicolumn{3}{|c|}{ Posterior/Anterior tilt $\left({ }^{\circ}\right)$} \\
\hline & & & IN & CON & ES & IN & CON & ES & IN & CON & ES \\
\hline & \multirow{3}{*}{30} & Before & $21.3 \pm 3.8$ & $26.1 \pm 5.6$ & \multirow{3}{*}{0.3} & $-3.7 \pm 6.1$ & $-3.7 \pm 3.4$ & \multirow{3}{*}{0.1} & $-4.5 \pm 5.6$ & $-2.9 \pm 5.0$ & \multirow{3}{*}{0.3} \\
\hline & & After & $20.7 \pm 4.0$ & $25.8 \pm 6.7$ & & $-3.6 \pm 7.0$ & $-3.4 \pm 3.7$ & & $-3.6 \pm 6.3$ & $-2.4 \pm 5.6$ & \\
\hline & & Change & $-1.1 \pm 2.9$ & $-0.3 \pm 2.1$ & & $0.1 \pm 2.5$ & $0.3 \pm 0 . .9$ & & $0.9 \pm 1.4$ & $0.5 \pm 0.9$ & \\
\hline & \multirow{3}{*}{40} & Before & $21.0 \pm 3.6$ & $25.6 \pm 5.7$ & \multirow{3}{*}{0.5} & $-7.5 \pm 6.1$ & $-7.9 \pm 3.8$ & \multirow{3}{*}{0.1} & $-3.5 \pm 5.8$ & $-1.8 \pm 5.0$ & \multirow{3}{*}{0.3} \\
\hline & & After & $19.6 \pm 6.7$ & $25.3 \pm 6.8$ & & $-7.6 \pm 7.1$ & $-7.7 \pm 4.0$ & & $-2.7 \pm 6.4$ & $-1.3 \pm 5.7$ & \\
\hline & & Change & $-1.4 \pm 2.9 *$ & $-0.3 \pm 1.8$ & & $-0.1 \pm 2.8$ & $0.3 \pm 0.9$ & & $0.8 \pm 1.5$ & $0.5 \pm 1.0$ & \\
\hline & \multirow{3}{*}{50} & Before & $20.9 \pm 3.5$ & $25.0 \pm 5.5$ & \multirow{3}{*}{0.5} & $-12.1 \pm 6.0$ & $-12.6 \pm 4.1$ & \multirow{3}{*}{0.1} & $-2.2 \pm 5.8$ & $-0.6 \pm 5.2$ & \multirow{3}{*}{0.7} \\
\hline & & After & $19.2 \pm 4.0$ & $24.7 \pm 6.8$ & & $-12.3 \pm 7.0$ & $-12.6 \pm 4.4$ & & $-0.9 \pm 6.5$ & $-0.1 \pm 5.8$ & \\
\hline & & Change & $-1.6 \pm 2.9 *$ & $-0.3 \pm 2.0$ & & $-0.1 \pm 3.0$ & $0.1 \pm 1.2$ & & $1.2 \pm 1.3^{*}$ & $0.4 \pm 1.0$ & \\
\hline $\mathrm{E}$ & \multirow{3}{*}{60} & Before & $20.8 \pm 3.5$ & $24.4 \pm 5.7$ & \multirow{3}{*}{0.6} & $-17.0 \pm 6.0$ & $-17.6 \pm 4.1$ & \multirow{3}{*}{0.2} & $-0.8 \pm 6.1$ & $0.9 \pm 5.0$ & \multirow{3}{*}{0.8} \\
\hline 1 & & After & $18.9 \pm 4.4$ & $24.2 \pm 6.9$ & & $-17.3 \pm 7.3$ & $-17.9 \pm 4.5$ & & $0.5 \pm 6.8$ & $1.3 \pm 5.8$ & \\
\hline $\begin{array}{l}e \\
v\end{array}$ & & Change & $-1.9 \pm 3.2 \dagger$ & $-0.2 \pm 2.2$ & & $-0.3 \pm 3.5$ & $-0.3 \pm 1.5$ & & $1.3 \pm 1.4^{*}$ & $0.3 \pm 1.3$ & \\
\hline$a$ & \multirow{3}{*}{70} & Before & $20.8 \pm 3.5$ & $23.5 \pm 5.8$ & \multirow{3}{*}{0.6} & $-21.8 \pm 5.9$ & $-22.4 \pm 4.3$ & \multirow{3}{*}{0.1} & $0.6 \pm 6.3$ & $2.6 \pm 5.2$ & \multirow{3}{*}{0.7} \\
\hline i & & After & $18.5 \pm 4.8$ & $23.0 \pm 7.3$ & & $-22.4 \pm 7.4$ & $-22.8 \pm 5.0$ & & $2.0 \pm 6.9$ & $3.1 \pm 5.8$ & \\
\hline 0 & & Change & $-2.3 \pm 3.4 \dagger$ & $-0.5 \pm 2.3$ & & $-0.6 \pm 3.8$ & $-0.4 \pm 1.6$ & & $1.5 \pm 1.6^{*}$ & $0.4 \pm 1.0$ & \\
\hline $\mathrm{a}$ & \multirow{3}{*}{80} & Before & $20.3 \pm 4.0$ & $22.8 \pm 6.2$ & \multirow{3}{*}{0.7} & $-26.2 \pm 11.1$ & $-26.9 \pm 4.8$ & \multirow{3}{*}{$<0.1$} & $2.2 \pm 6.5$ & $4.4 \pm 5.3$ & \multirow{3}{*}{1.0} \\
\hline $\begin{array}{l}n \\
g\end{array}$ & & After & $17.7 \pm 5.5$ & $22.4 \pm 7.4$ & & $-26.9 \pm 7.7$ & $-27.6 \pm 5.5$ & & $4.3 \pm 7.3$ & $4.9 \pm 5.9$ & \\
\hline $\begin{array}{l}\text { o } \\
1\end{array}$ & & Change & $-2.6 \pm 3.4 \dagger$ & $-0.4 \pm 2.3$ & & $-0.7 \pm 3.9$ & $-0.7 \pm 1.6$ & & $2.1 \pm 1.8^{*}$ & $0.5 \pm 1.2$ & \\
\hline $\begin{array}{l}\text { e } \\
(\end{array}$ & & Before & $20.1 \pm 4.5$ & $22.2 \pm 6.6$ & & $-30.1 \pm 6.0$ & $-30.9 \pm 5.2$ & & $3.8 \pm 6.7$ & $6.2 \pm 5.4$ & \\
\hline o & 90 & After & $17.0 \pm 6.6$ & $21.6 \pm 7.7$ & 0.7 & $-31.0 \pm 8.0$ & $-31.7 \pm 6.1$ & $<0.1$ & $6.6 \pm 7.7$ & $6.8 \pm 6.0$ & 1.1 \\
\hline ) & & Change & $-3.0 \pm 3.9 \dagger$ & $-0.6 \pm 2.3$ & & $-0.9 \pm 4.0$ & $-0.8 \pm 1.4$ & & $2.8 \pm 2.2 \dagger$ & $0.7 \pm 1.3$ & \\
\hline & & Before & $20.1 \pm 5.0$ & $21.7 \pm 7.1$ & & $-33.6 \pm 6.1$ & $-34.2 \pm 5.6$ & & $5.5 \pm 6.7$ & $7.9 \pm 5.5$ & \\
\hline & 100 & After & $16.8 \pm 6.9$ & $21.2 \pm 8.0$ & 0.8 & $-34.6 \pm 8.3$ & $-35.3 \pm 6.8$ & $<0.1$ & $8.8 \pm 8.1$ & $8.9 \pm 6.2$ & 1.0 \\
\hline & & Change & $-3.3 \pm 4.1 \dagger$ & $-0.5 \pm 2.3$ & & $-1.0 \pm 4.3$ & $-1.0 \pm 1.5$ & & $3.3 \pm 2.6 *$ & $1.0 \pm 1.5$ & \\
\hline & & Before & $20.1 \pm 5.8$ & $21.4 \pm 7.7$ & & $-37.1 \pm 6.3$ & $-37.3 \pm 6.1$ & & $7.1 \pm 6.6$ & $9.2 \pm 5.7$ & \\
\hline & 110 & After & $16.3 \pm 8.0$ & $20.5 \pm 8.8$ & 0.8 & $-38.1 \pm 8.4$ & $-38.3 \pm 7.1$ & $<0.1$ & $10.9 \pm 8.4$ & $10.5 \pm 6.6$ & 0.9 \\
\hline & & Change & $-3.8 \pm 4.3 \dagger$ & $-0.8 \pm 2.4$ & & $-1.0 \pm 4.4$ & $-1.0 \pm 1.3$ & & $3.8 \pm 3.0^{*}$ & $1.3 \pm 1.6$ & \\
\hline & & Before & $20.4 \pm 6.6$ & $21.5 \pm 8.9$ & & $-40.4 \pm 6.6$ & $-40.1 \pm 6.5$ & & $8.3 \pm 6.4$ & $10.1 \pm 6.1$ & \\
\hline & 120 & After & $15.9 \pm 8.6$ & $20.1 \pm 10.2$ & 0.7 & $-41.0 \pm 8.9$ & $-41.1 \pm 7.6$ & 0.1 & $12.0 \pm 8.3$ & $11.5 \pm 7.4$ & 0.8 \\
\hline & & Change & $-4.5 \pm 4.9 \dagger$ & $-1.4 \pm 2.5$ & & $-0.6 \pm 4.7$ & $-1.0 \pm 1.4$ & & $3.7 \pm 3.1^{*}$ & $1.5 \pm 1.8$ & \\
\hline
\end{tabular}

501

502

503

504

505
IN, interventional limb; CON, control limb; ES, effect size of amount of change; Before, raw value before stretching; After, raw value after stretching; Change, amount of change between before and after stretching. Values are expressed as mean \pm standard deviation. The asterisk indicates that the change in scapular motion in the interventional limb is significantly $(P<.05)$ greater than it in the control limb; the single dagger 
Scapular kinematics and pectoralis minor stiffness

506

indicates that it in the interventional limb is significantly $(P<.01)$ greater than it in the control limb.

507

508 